\title{
REPRODUTIBILIDADE INTRA-OBSERVADOR DE UM PROTOCOLO PARA AVALIAÇÃO POSTURAL EM ESCOLARES ${ }^{1}$
}

\section{Juliana Adami Sedrez}

Universidade Federal do Rio Grande do Sul, Porto Alegre, Rio Grande do Sul, Brasil

\section{Daniela Scotto de Oliveira}

Universidade do Vale do Rio do Sinos, São Leopoldo, Rio Grande do Sul, Brasil

\section{Matias Noll}

Universidade Federal do Rio Grande do Sul, Porto Alegre, Rio Grande do Sul, Brasil

\section{Cíntia Detsch Fonseca}

Universidade Federal do Rio Grande do Sul, Porto Alegre, Rio Grande do Sul, Brasil

\section{Cláudia Tarragô Candotti}

Universidade Federal do Rio Grande do Sul, Porto Alegre, Rio Grande do Sul, Brasil

\section{Resumo}

O objetivo do estudo foi propor e verificar a reprodutibilidade intra-observador de um protocolo de avaliação postural para o ambiente escolar. Vinte escolares foram submetidos a um protocolo de avaliação postural, com registro fotográfico, avaliação da gibosidade e medição das alturas dos acrômios e das espinhas ilíacas póstero-superiores. As avaliações foram realizadas em teste e re-teste, com intervalo de 14 dias. Os resultados no plano frontal foram moderados para a horizontalidade da pelve e ombro e excelentes para gibosidade e tipo de escoliose. No plano sagital, os índices foram moderados para postura da pelve, ombro, cabeça e tipo de postura. A metodologia proposta pode ser utilizada em ambiente escolar, visto que possui bons índices de reprodutibilidade intra-observador.

Palavras-chave: Postura. Avaliação. Saúde Escolar.

\section{Introdução}

tualmente na literatura encontram-se diferentes definições de
ticos e em outras os aspectos biomecânicos, mas de forma consensual,

1-O presente trabalho não contou com apoio financeiro de nenhuma natureza para sua realização 
acredita-se que ambos os aspectos influenciam no arranjo postural. Considerando que o termo postura corporal pode ser entendido como a posição que o corpo assume no espaço de forma a buscar o equilíbrio de suas estruturas (DETSCH; CANDOTTI, 2001), entende-se que a manutenção da postura corporal depende de uma complexa interação de mecanismos fisiológicos, os quais sofrem influências centrais e periféricas para garantir ao indivíduo a capacidade de ficar em pé, andar e interagir com o meio ambiente de forma segura e eficiente (MOREIRA, 2008).

Durante a fase de crescimento, na transição entre infância e idade adulta, a postura da criança sofre transformações na busca de um equilíbrio compatível com as novas proporções de seu corpo, além disso, nessa idade, a mobilidade é extrema, e, portanto a postura tende a adaptar-se facilmente (FERRONATTO; CANDOTTI; SILVEIRA, 1998). Não obstante, se forem mantidas posturas inadequadas, podese comprometer o equilíbrio corporal, o qual se encontra justamente em uma fase de organização, acarretando alterações futuras na fase adulta. (FERRONATTO; CANDOTTI; SILVEIRA, 1998) Esse, sem dúvida, é um importante motivo para a realização de observação e identificação precoce dos desvios posturais acentuados ou persistentes nos indivíduos em crescimento (DETSCH; CANDOTTI, 2001).

No entanto, para tornar viável a prática de avaliações posturais sistemáticas no ambiente escolar torna-se necessário o acesso a metodologias simples, econômicas e de fácil utilização. Nesse sentido, a clássica avaliação da postura, baseada na inspeção visual, que utiliza um fio de prumo e um posturógrafo, têm sido bem aceitos na literatura, sendo utilizados tanto em estudos de incidência e prevalência de desvios posturais (DETSCH et al., 2007; SANTOS et al., 2009; FERRONATTO; CANDOTTI; SILVEIRA, 1998; DETSCH; CANDOTTI, 2001; MARTELLI; TRAEBERT, 2006), quanto em estudos de intervenção, que utilizam a metodologia das escolas posturais (CANDOTTI et al., 2009; CANDOTTI et al., 2010). Apesar desta aceitação, chama à atenção a falta de evidências científicas sobre a validade e reprodutibilidade de um protocolo de avaliação postural baseada na inspeção visual, caracterizando assim, uma lacuna na literatura. Desse modo, o objetivo do presente estudo foi propor um protocolo de avaliação postural para o ambiente escolar e testar a sua reprodutibilidade intra-observador. 


\section{Metodologia}

A amostra foi constituída de 20 escolares do sexo feminino, com idades entre 12 e 13 anos que frequentavam a sétima série do nível fundamental de uma escola da cidade de Novo Hamburgo, Rio Grande do Sul. O estudo foi aprovado pelo Comitê de Ética em Pesquisa da Universidade em que está vinculado. Os escolares receberam um termo de consentimento livre e esclarecido para leitura, decisão de participação e assinatura dos responsáveis.

Os escolares foram submetidos a um protocolo de avaliação postural estática e a medição da estatura e da massa corporal. Para as avaliações foram utilizadas roupas apropriadas (corsário, top ou biquíni), cabelo preso e pés descalços. O protocolo de avaliação postural estática foi realizado em dois dias distintos, com intervalo de 14 dias entre eles, mantendo constante todas as demais condições (local, horário e avaliador) com objetivo verificar a reprodutibilidade intraobservador.

Protocolo de avaliação postural estática

O protocolo proposto no presente estudo foi desenvolvido com base nos estudos de Candotti, Zatti e Halmerski (2005), Detsch et al. (2007) e Ferronato, Candotti e Silveira (1998), sendo composto por: (1) observação da postura estática nos planos frontal e sagital, a partir de fotografias (Figura 1); (2) medições de alturas das escápulas, dos acrômios e das espinhas ilíacas póstero-superiores (EIPS) e (3) avaliação da presença de gibosidade.

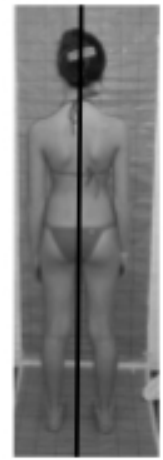

(a)

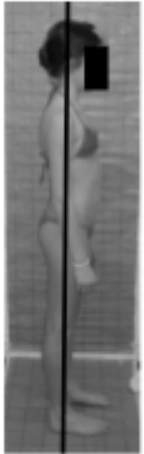

(b)

Figura 1: Avaliação postural utilizando o posturógrafo: (a) no plano frontal, (b) no plano sagital. Ilustração do fio de prumo traçado sobre cada imagem 
Os materiais utilizados para a coleta dos dados (posturógrafo, máquina fotográfica, antropômetro adaptado a uma trena retrátil e ficha de dados) podem ser facilmente produzidos pelo avaliador, tornando a metodologia de baixo custo, possibilitando sua aplicação em locais sem estrutura apropriada para a realização de outros protocolos de avaliação postural.

Para a observação da postura estática no plano frontal e no plano sagital direito (Figura 1), o indivíduo foi posicionado à frente de um posturógrafo, o qual era confeccionado de papel pardo, com dimensões de 2,00 x 0,60 m e com quadriculados a cada $0,10 \mathrm{~m}$, e, era fixado na parede, sendo utilizado um nível a fim de garantir a horizontalidade das linhas. Além disso, foi utilizada uma base para os pés, com dimensões de $0,60 \times 0,60$, com quadriculado idêntico ao do posturógrafo. Foram realizados registros fotográficos com uma máquina fotográfica digital (Olympus D-390, e megapixels) afixada sobre um tripé a uma altura de $1,48 \mathrm{~m}$ e a uma distância de 2,00 $\mathrm{m}$ do posturógrafo.

Para a medição das alturas dos acrômios (Figura 2a) e das EIPS (Figura 2b), o indivíduo se posicionou de costas para a avaliadora, distribuindo o peso corporal sobre os dois pés. Foram palpados e marcados os seguintes pontos anatômicos: acrômio direito e esquerdo, ângulos inferiores das escápulas direita e esquerda e EIPS direita e esquerda. Após foi utilizado um antropômetro adaptado a uma trena retrátil, com nível acoplado na haste do antropômetro. Esta medida foi obtida colocando-se a haste do antropômetro no ponto anatômico marcado e o zero da fita métrica no chão (Figura 2) a fim de verificar a horizontalidade dos ombros e a horizontalidade da pelve, ou seja, a simetria das alturas entre os lados direito e esquerdo a partir do acrômio e das EIPS, respectivamente.

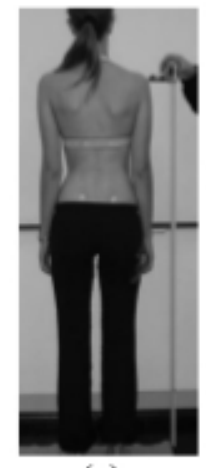

(a)

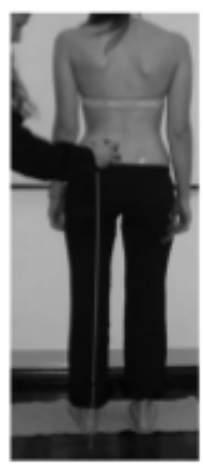

(b)

Figura 2: Medição das alturas: (a) acrômio direito e EIPS esquerda

Pensar a Prática, Goiânia, v. 16, n. 2, p. 320-618, abr./jun. 2013 
Para avaliação da gibosidade, foi realizado o teste de Adams, para o qual o indivíduo era posicionado de costas para a avaliadora, com os pés unidos em paralelo, os joelhos estendidos e o olhar na linha do horizonte. A seguir era solicitado que o indivíduo flexionasse o tronco, mantendo seus braços pendentes com as palmas das mãos unidas (NUSSINOVITCH et al., 2002). A partir desta posição, era solicitado que o indivíduo fizesse lentamente a extensão do tronco, enquanto a avaliadora observava a presença ou ausência de gibosidade.

Procedimentos de análise das fotografias

A análise da postura estática foi realizada através da observação das fotografias, nos dois planos: frontal e sagital. Para isso, sobre as fotografias nas quais o indivíduo se encontra de costas foi traçada uma linha vertical (fio de prumo) a partir de um ponto equidistante entre calcanhares. Os pontos de referência padrão que serviram para perfilar a vista posterior da postura estática dos indivíduos foram: (1) através da linha média da pelve; (2) através da coluna vertebral; (3) dividindo a cabeça em duas partes iguais (KENDALL; McREARY; PROVANCE, 1995).

A observação das fotografias no plano frontal permitiu a avaliação da posição dos ombros, das escápulas e da coluna vertebral. A informação sobre a posição dos ombros e da escápula oriunda das fotografias foi utilizada para confirmação dos dados sobre a horizontalidade do ombro, obtidos pela medição das alturas dos acrômios direito e esquerdo.

A postura da coluna vertebral, no plano frontal, dos indivíduos foi classificada em quatro tipos: (1) coluna normal, sem qualquer escoliose; (2) coluna com postura de escoliose, com curvatura convexa à esquerda; (3) coluna com postura de escoliose, com curvatura convexa à direita; (4) coluna com postura de escoliose em "S". Para esta classificação foram utilizados os dados obtidos com as medições das alturas (EIPS e acrômio) e com o teste de Adams (observação da gibosidade). De posse destes dados foi construído um desenho esquemático da postura dos indivíduos (Figura 3), o qual permitiu identificar o padrão da escoliose de cada indivíduo (CANDOTTI; ZATTI; HALMERSKI, 2005), utilizando o referencial proposto por Kendall, Mcreary e Provance (1995): (a) o lado em que a pelve está mais baixa corresponde ao lado convexo da curvatura, (b) a gibosidade está localizada no lado 
convexo da curvatura, (c) se a pelve e o acrômio estiveram mais elevados no mesmo lado, provavelmente existe uma escoliose em "S" e (d) se a pelve e o acrômio estiveram mais elevados em lados contrários, provavelmente existe uma escoliose em "C". A informação sobre a posição da coluna vertebral, oriunda da observação das fotografias no plano frontal foi utilizada apenas para confirmação dos dados obtidos pela medição das alturas dos acrômios e EIPS.

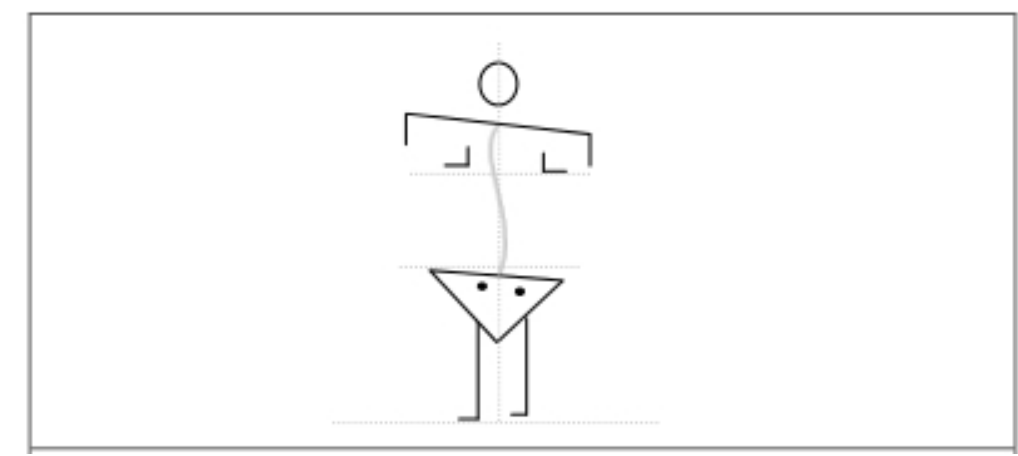

Figura 3: Exemplo de desenho esquemático da postura

Para a análise das fotografias no plano sagital foi tracejado um fio de prumo a partir da fossa anterior do maléolo lateral. Os pontos de referência padrão que serviram para perfilar lateralmente a postura estática dos indivíduos foram: (1) fossa anterior do maléolo lateral; (2) eixo da articulação do quadril; (3) articulação do ombro e (4) meato auditivo externo (KENDALL; McREARY; PROVANCE, 1995). A observação das fotografias no plano sagital permitiu a avaliação da posição da cabeça, dos ombros, da pelve e da coluna vertebral. A análise das fotografias, no plano sagital, permitiu a seguinte classificação: (a) quanto à posição da cabeça: normal, anteriorizada ou retraída; (b) quanto ao posicionamento dos ombros: normal, protusos ou retraídos; e (c) quanto ao posicionamento da pelve: normal, antevertida ou retrovertida. A postura da coluna vertebral, no plano sagital, dos indivíduos foi classificada em quatro tipos, segundo Kendal, McReary e Provance (1995): (1) postura no alinhamento ideal; (2) postura de cifose-lordose; (3) postura de dorso plano e (4) postura desleixada.

Tratamento estatístico

Os resultados do protocolo de avaliação postural realizados nos dois dias de avaliação foram comparados utilizado o software SPSS 18.0. Inicialmente foi verificada a equivalência das variâncias (teste 
de Levene) e normalidade dos dados (Shapiro-Wilk). Os dados foram submetidos a um teste de correlação intra-classe (ICC). Os valores de ICC encontrados foram classificados em fracos (ICC $<0,40$ ), moderados (ICC entre 0,4 e 0,75 e excelentes (ICC >0,75), de acordo com Fleiss (1986). O nível de significância adotado foi 0,05 .

\section{Resultados}

Os resultados deste estudo indicam que o protocolo de avaliação postural proposto para ambiente escolar apresentou moderada e excelente reprodutibilidade intra-avaliador (Tabelas 1 e 2) para as variáveis analisadas no plano frontal (horizontalidade do ombro e pelve, gibosidade e tipo de escoliose) e no plano sagital (posição da cabeça, ombros e pelve e tipo de postura da coluna vertebral).

Na Tabela 1 são apresentados os coeficientes de correlação entre teste e re-teste para as variáveis observadas no plano frontal.

Tabela 1: Resultados obtidos com o de Teste de Correlação Intra-Classe entre o teste e re-teste, para as variáveis avaliadas no plano frontal

\begin{tabular}{l|c|c}
\cline { 2 - 3 } & $\begin{array}{c}\text { Coeficiente de } \\
\text { correlação } \\
\text { (ICC) }\end{array}$ & Valor p \\
\hline Horizontalidade da pelve & 0,546 & $0,035^{*}$ \\
\hline Horizontalidade do ombro & 0,707 & $0,003^{*}$ \\
\hline Gibosidade & 0,764 & $0,001^{*}$ \\
\hline Tipo de escoliose & 0,972 & $0,000^{*}$ \\
\hline
\end{tabular}

*correlação significativa: $\mathrm{p}<0,05$

Na Tabela 2 são apresentados os coeficientes de correlação entre teste e re-teste para as variáveis observadas no plano sagital.

Tabela 2: Resultados obtidos com o Teste de Correlação Intra-Classe entre o teste e re-teste, para as variáveis avaliadas no plano sagital

\begin{tabular}{|c|c|c|}
\hline & $\begin{array}{c}\text { Coeficiente de } \\
\text { correlação (ICC) }\end{array}$ & Valor $\mathrm{p}$ \\
\hline Posição da cabeça & 0,739 & $0,002^{*}$ \\
\hline Posição do ombro & 0,706 & $0,003 *$ \\
\hline Posição da pelve & 0,642 & $0,010^{*}$ \\
\hline Tipo de postura da coluna & 0,701 & $0,004 *$ \\
\hline
\end{tabular}

*correlação significativa: $\mathrm{p}<0,05$ 


\section{Discussão}

Esse estudo objetivou propor um protocolo de avaliação postural para o ambiente escolar e testar a sua reprodutibilidade intra-observador. Os resultados demonstram moderada a excelente correlação intraclasse entre os resultados obtidos nos dois dias de avaliação, evidenciando a reprodutibilidade intra-observador deste protocolo de avaliação postural.

Segundo Seah et al. (2011) a avaliação da postura corporal apresenta grande importância clínica, sendo que a classificação postural parece ser clinicamente mais relevante do que apenas a informação de ângulos distintos para a análise da postura da coluna vertebral em geral. Seah et al. (2011) demonstraram que a avaliação clínica postural apresentou de moderada a boa confiabilidade inter-observador. No entanto estes resultados se referem a uma comparação dos resultados de dois avaliadores apenas no que tange o padrão postural dos indivíduos avaliados e não durante a utilização de um protocolo específico, como no presente estudo.

Na literatura também é possível observar a avaliação da reprodutibilidade intra-observador em diferentes métodos de avaliação postural de caráter quantitativo, especialmente no plano sagital. Por exemplo, no estudo de Hart e Rose (1986) foi realizada a reprodutibilidade intra-observador utilizando o flexicurva para a avaliação da curvatura lombar em oitenta e nove sujeitos, sendo observadas duas posturas, em pé e em completa flexão anterior, as quais apresentaram boa correlação no teste-reteste $(\mathrm{ICC}=0,97)$, demonstrando a reprodutibilidade do instrumento flexicurva na avaliação da coluna lombar. Outro exemplo é o estudo de D’Osualdo, Schierano e Iannis (1997), que também encontrou boas correlações para as avaliações realizadas com uma semana de intervalo utilizando o instrumento arcômetro na medição da curvatura torácica, demonstrando que o instrumento possibilita reprodutibilidade intra-observador.

Tal como a maioria dos estudos que apresentam instrumentos para avaliação no plano sagital, o presente estudo também demonstrou que o protocolo de avaliação postural proposto é reprodutível, sendo encontrados resultados moderados e significativos para posição da pelve, posição do ombro, posição da cabeça e tipo de postura da coluna vertebral (Tabela 2). A principal diferença entre esses estudos reside no fato da subjetividade da avaliação postural. Os instrumentos flexi- 
curva (HART; ROSE, 1986) e arcomêtro (D'OSUALDO; SCHIERANO; IANNIS, 1997) propõem justamente eliminar esta questão, fornecendo valores em graus das curvaturas da coluna vertebral. Entretanto, estes instrumentos limitam-se a avaliação das curvaturas no plano sagital, não fornecendo qualquer outra informação acerca das posições dos demais segmentos corporais.

Neste sentido, é importante salientar que em diversos instrumentos que realizam avaliação postural quantitativa, é realizada somente a avaliação um plano. Nesse sentido, são necessários estudos a fim de aperfeiçoar e desenvolver novas metodologias que forneçam informações mais globais da postura. Sendo assim, diferentemente da maioria dos estudos (HART; ROSE, 1986; D'OSUALDO; SCHIERANO; IANNIS, 1997; OHLÉN; SPANGFORT; TINGVALL, 1989), o presente protocolo de avaliação da postura também possibilita a avaliação no plano frontal. Os resultados demonstraram boa reprodutibilidade intra-observador para as variáveis avaliadas, sendo encontradas correlações significativas e moderadas para a horizontalidade da pelve e horizontalidade do ombro e correlações significativas e excelentes para gibosidade e tipo de escoliose (Tabela 1). Portanto, o protocolo de avaliação postural proposto no presente estudo, embora não forneça informações quantitativas das curvaturas da coluna, permite uma avaliação mais ampla da postura corporal, as quais evidenciam as assimetrias entre os lados direito e esquerdo do corpo, facilitando a análise da postura também no plano frontal.

Outro aspecto que deve ser considerado é que independentemente do instrumento ou protocolo utilizado, a avaliação da postura é intimamente dependente da experiência do examinador, já que para uma correta avaliação se faz necessário primeiramente uma apropriada marcação dos pontos anatômicos de referência. Por esse motivo, uma palpação adequada está fortemente relacionada ao sucesso na avaliação (HARLICK; MILOSAVLJEVIC; MILBURN, 2007; HANELINE; YOUNG, 2009). Além disso, estudos têm ressaltado alguns fatores que podem aumentar a dificuldade da palpação, como por exemplo, maiores índices de massa corporal (HARLICK; MILOSAVLJEVIC; MILBURN, 2007; DEGENHARDT et al., 2005, TROKE; SCHUIT; PETERSEN, 2007). Sendo assim, para a reprodução dos resultados desse estudo, se torna necessária a realização adequada do protocolo, para o qual é necessário que o observador tenha experiência em pal- 
pação e esteja apto para a realização da palpação dos pontos anatômicos de forma correta.

No estudo de Furlanetto et al. (2011) foi proposta uma metodologia para avaliação postural composta por (1) palpação e marcação de pontos anatômicos, (2) registro fotográfico nos planos frontal e sagital e (3) digitalização dos pontos anatômicos no software APPID (Avaliação Postural a Partir de Imagem Digital). Os resultados de desse estudo demonstraram correlações significativas para a totalidade das variáveis estudadas, tais como curvatura cervical (ICC $=0,742$ ), curvatura dorsal $(\mathrm{ICC}=0,964)$, curvatura lombar $(\mathrm{ICC}=0,666)$ e curvatura escoliótica $(\mathrm{r}=0,985)$, indicando que a metodologia proposta apresenta reprodutibilidade intra-observador nos planos frontal e sagital. Duas importantes diferenças podem ser apontadas entre o método proposto por Furlanetto et al. (2011) e o protocolo proposto no presente estudo: (1) no primeiro estudo a analise das fotografias é realizada por um software, enquanto que no presente estudo é realizada manualmente, por observação; e (2) somente o primeiro estudo fornece valores quantitativos sobre as curvaturas da coluna vertebral.

Outro estudo, de Rocha e Souza (1999) realizado com dez indivíduos trabalhadores da indústria, também avaliou a reprodutibilidade intra-observador de um protocolo de avaliação postural dinâmica, através de filmagens de seis movimentos cotidianos. Os autores encontram alta correlação entre as avaliações e concluíram que o instrumento permite avaliação reprodutível da postura dinâmica.

A literatura apresenta inúmeros métodos, protocolos ou instrumentos para avaliação da coluna vertebral ou da postura corporal estática e dinâmica, mas a quase totalidade deles não visa o público infanto-juvenil no contexto da escola. Dentre os que envolvem a avaliação da postura no ambiente escolar, cita-se o estudo de Ritter e Souza (2006) que reportou reprodutibilidade intra-observador para um instrumento que avalia a percepção de alunos sobre sua postura adotada no dia-adia. Esse instrumento consiste em um questionário, o qual foi respondido pelos escolares em dois dias distintos, com um intervalo de 10 dias entre eles. Os resultados mostraram correlação alta e significativa $(\mathrm{r}=0,91 ; \mathrm{p}<0,000)$ entre as duas avaliações. De maneira semelhante, o presente estudo apresentou boa correlação entre o teste e re-teste, assegurando a sua reprodutibilidade intra-observador, demonstrando que a metodologia proposta, que envolve o registro fotográfico da postura ereta nos planos frontal e sagital, a medição das alturas da EIPS e 
acrômio e a avaliação da gibosidade, pode ser adotada no ambiente escolar, auxiliando na detecção precoce de alterações posturais.

No estudo de Graup, Santos e Moro (2010), realizado com escolares, $53,8 \%$ dos sujeitos apresentavam algum desvio postural na coluna lombar, sendo $90,9 \%$ apresentavam retificação da coluna lombar e $9,1 \%$, hiperlordose lombar, demonstrando que, mesmo em indivíduos mais jovens já são encontradas elevadas prevalências de alterações posturais. Esses mesmos autores encontraram uma elevada prevalência de dor lombar, a qual foi relatada por $49,6 \%$ dos escolares, o que demonstra a necessidade de avaliar precocemente essa população, a fim de possibilitar a identificação precoce de alterações posturais no âmbito escolar.

Segundo Rego e Scartoni (2008), durante o período escolar, se forem adotadas posturas assimétricas prolongadas pode-se acarretar em alterações posturais, as quais se persistirem, podem gerar desconforto, dor ou incapacidade. Tais alterações poderiam ser prevenidas através da avaliação postural precoce, educação postural e adequação do mobiliário escolar. Esses mesmos autores sugerem a adoção da avaliação postural e educação postural como medidas preventivas no âmbito escolar, com a finalidade de evitar que as crianças desenvolvam futuramente deformidades estruturais. Ainda, segundo Seah et al. (2011), uma melhor compreensão da postura e seus fatores relacionados podem permitir um melhor entendimento sobre a saúde da coluna vertebral.

Considerando que, de modo geral, a literatura tem consistentemente demonstrado a importância da avaliação postural no ambiente escolar, entende-se que o protocolo de avaliação postural proposto no presente estudo, por ser de fácil aplicação, consiste em uma importante ferramenta para detecção precoce de alterações posturais, e pode auxiliar a prática dos profissionais de educação física inseridos no âmbito escolar.

\section{Conclusão}

Os resultados demonstraram que a metodologia proposta para avaliação postural no plano frontal e sagital em ambiente escolar pode ser efetivamente utilizada, visto que possui bons índices de reprodutibilidade intra-observador, é de fácil utilização e análise, consistindo em 
uma importante ferramenta na deteç̧ão precoce de alterações posturais.

Intraobserver reproducibility of a protocol for evaluation postural in students

\begin{abstract}
The study objective was to propose and verify the intraobserver reproducibility of a postural assessment protocol for the school environment. Twenty children were subjected to a postural assessment protocol, with photographic recording and evaluation of spinal deformity and measuring the heights of the acromion and the posterior superior iliac spines. The evaluations were conducted in test-retest at an interval of 14 days. The results in the frontal plane were moderate to the horizontality of the pelvis and shoulder and excellent for type of scoliosis and spinal deformity. In the sagittal plane, the indexes were moderate approach to the pelvis, shoulder, head and kind of attitude. The proposed methodology can be used in the school environment, which has seen good levels of intra-observer reproducibility.
\end{abstract}

Keywords: Posture. Evaluation. School Health.

\title{
Reproducibilidad intra-observador de um protocolo para la evaluación pos- tural en los estudiantes
}

\section{Resumen}

El objetivo del estudio fue proponer y verificar la reproducibilidad intraobservador de un protocolo de evaluación postural para el ambiente escolar. Veinte escolares fueron sometidos a un protocolo de evaluación postural con registro fotográfico, evaluación de la gibosidad y medición de las alturas de los acromiones y de las espinas ilíacas posterosuperiores. Las evaluaciones fueron realizadas en test-retest con intervalo de catorce días. Los resultados en el plano frontal fueron moderados para la horizontalidad de la pelvis y del hombro y, excelentes para gibosidad y tipo de escoliosis. En el plano sagital, los índices fueron moderados para postura de pelvis, hombro, cabeza y tipo de postura. La metodología propuesta puede ser utilizada en ambiente escolar, ya que posee buenos índices de reproducibilidad intraobservador.

Palabras clave: Postura. Evaluación. Salud Escolar.

\section{Referências}

CANDOTTI, C. T. et al. Escola de postura: uma metodologia adaptada aos pubescentes. Revista Mackenzie de Educação Física e Esporte, São Paulo, v. 9, n. 2, p. 91-100, 2010.

CANDOTTI, C. T. et al. Escola postural: uma metodologia adaptada para crianças. Arquivos em Movimento, Rio de Janeiro, v. 5, n. 2, p. 34-49, 2009. 
CANDOTTI, C. T.; ZATTI, V.; HALMERSKI, C. Proposição de um método indireto para avaliação clínica da escoliose. Ação \& Movimento, v. 2, n. 6, p. 313-9, nov./dez., 2005.

DEGENHARDT, B. F. et al. Interobserver reliability of osteopathic palpatory diagnostic tests of the lumbar spine: Improvements from consensus training. Journal of the American Osteopathic Association, v. 105, n. 10, p. 465-473, out., 2005.

DETSCH, C.; CANDOTTI, C. T. A incidência de desvios posturais em meninas de 6 a 17 anos da cidade de Novo Hamburgo. Movimento, Porto Alegre, v. 7, n. 15, p. 43-56, 2001.

DETSCH, C. et al. Prevalência de alterações posturais em escolares do ensino médio em uma cidade no Sul do Brasil. Revista Panamericana de Salud Pública, v. 21, n. 4, p. 231-8, 2007.

D'OSUALDO, F.; SCHIERANO, S.; IANNIS, M. Validation of Clinical Measurement of Kyphosis With a Simple Instrument, the Arcometer. Spine, v. 22, n. 4, p. 408-13, 1997.

FERRONATTO, A; CANDOTTI, C. T.; SILVEIRA, R. P. A incidência de alterações do equilíbrio estático da cintura escapular em crianças entre 7 a 14 anos. Movimento, Porto Alegre, v. 5, n. 9, p. 24-30, 1998.

FLEISS, R. L. The design and analysis of clinical experiments. New York: John Wiley and Sons, 1986.

FURLANETTO, T. $\mathrm{S}$ et al. Fidedignidade de um protocolo de avaliação postural. Revista da Educação Física/UEM, Maringá, v. 22, n. 3, p. 411-9, jul./set., 2011.

GRAUP, S.; SANTOS, S. G.; MORO, A. R. P. Estudo descritivo de alterações posturais sagitais da coluna lombar em escolares da rede federal de ensino de Florianópolis. Revista Brasileira de Ortopedia, São Paulo, v. 45, n. 5, p. 453-9, 2010.

HANELINE M. T.; YOUNG, M. A review of intraexaminer and interexaminer reliability of static spinal palpation: a literature synthesis. Journal of Manipulative and Physiological Therapeutics, v. 32, n. 5, p. 379-86, 2009. 
HARLICK, J. C.; MILOSAVLJEVIC, S.; MILBURN, P. D. Palpation identification of spinous processes in the lumbar spine. Manual Therapy, v. 12, n. 1, p. 56-62, 2007.

HART, D. L.; ROSE, S. Reliability of a noninvasive method for measuring the lumbar curve. The journal of Orthopaedic and Sports Physical Therapy, v. 8, n. 4, p. 180-4, 1986.

KENDALL, F. P.; MCREARY, E. K.; PROVANCE, P. G. Músculos provas e funções. 4. ed. São Paulo: Manole, 1995.

MARTELLI, R. C.; TRAEBERT, J. Estudo descritivo das alterações posturais de coluna vertebral em escolares de 10 a 16 anos de idade: Tangará-SC, 2004. Revista Brasileira de Epidemiologia, São Paulo, v. 9, n. 1, p. 87-93, mar., 2006.

MOREIRA, S. Características da postura corporal de escolares da rede municipal de ensino de Porto Alegre. 2008. 117 f. Dissertação (Mestrado em Educação Física)-Escola de Educação Física, UFRGS, Porto Alegre, 2008.

NUSSINOVITCH, $\mathrm{M}$ et al. Adolescent screening for orthopedic problems in high school. Public Health, v. 116, n. 1, p. 30-2, 2002.

OHLÉN, G.; SPANGFORT, E.; TINGVALL, C. Measurement of spinal sagittal configuration and mobility with Debrunner's kyphometer. Spine, v. 14, n. 6, p. 580-3, 1989.

REGO, A. R. O.; SCARTONI, F. R. Alterações posturais de alunos de $5^{\mathrm{a}}$ e $6^{\mathrm{a}}$ séries do ensino fundamental. Fitness \& Performance Journal, Rio de janeiro, v. 7, n. 1, p. 10-5, jan./fev., 2008.

RITTER, AL; DE SOUZA, JL. Instrumento para conhecimento da percepção de alunos sobre a postura adotada no ambiente escolar Posper. Movimento, Porto Alegre, v. 12, n. 3, p. 249-62, set./dez., 2006.

ROCHA, AS; SOUZA, JL. Observações das atividades de vida diária através de vídeo. Movimento, Porto Alegre, v. 5, n. 11, p. 16-22, mar., 1999. 
SANTOS, C. I. S. et al. Ocorrência de desvios posturais em escolares do ensino público fundamental de Jaguariúna, São Paulo. Revista Paulista de Pediatria, São Paulo, v. 27, n. 1, p. 74-80, mar., 2009.

SEAH, S. H. H. et al. An exploration of familial associations in spinal posture defined using a clinical grouping method. Manual Therapy, v. 16, n. 5, p. 501-9, 2011.

TROKE, R. M.; SCHUIT, D.; PETERSEN, C. M. Reliability of lumbar spinal palpation, range of motion, and determination of position. BMC Musculoskeletal Disorders, v. 8, p. 103, 2007.

Recebido em: 11/01/2012

Revisado em: 08/06/2012

Aprovado em: 29/08/2012

Endereço para correspondência

claudia.candotti@ufrgs.br

Cláudia Tarragô Candotti

Universidade Federal do Rio Grande do Sul

Escola Superior de Educação Física.

Rua Felizardo, 750

Jardim Botânico

90690-200 - Porto Alegre, RS - Brasil 